\title{
La communauté de pratique
} un outil pertinent : résumé des connaissances adaptées au contexte de la santé publique

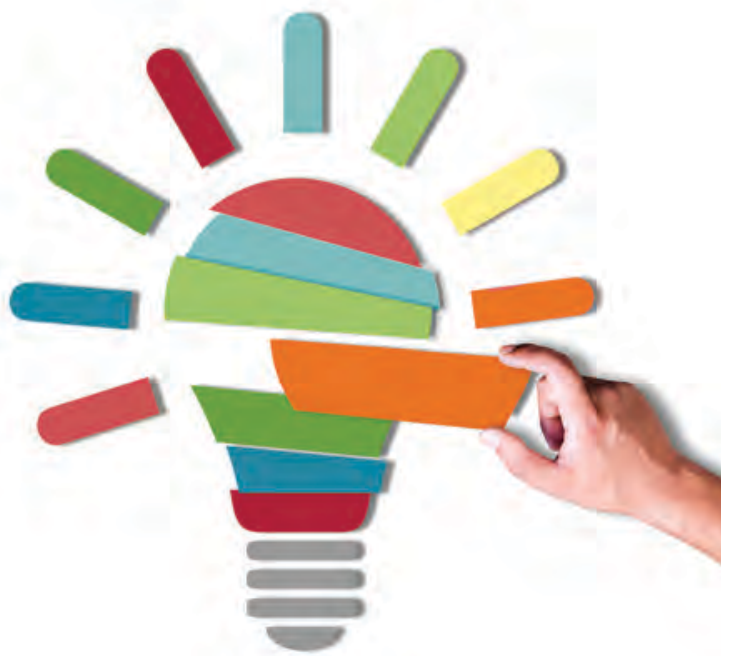

\section{FICHE D'INFORMATION}

La présente fiche vise à répondre aux besoins d'informations exprimés par des personnes consultées dans le cadre d'une démarche exploratoire menée par l'INSPQ : besoins d'informations factuelles sur ce qu'est ou n'est pas une communauté de pratique, ses avantages, limites, indications et implications en santé publique.

\section{Sommaire}

Une communauté de pratique, de quoi parle-t-on?

Comment fonctionne une communauté de pratique?

Quelles sont les étapes de mise en œuvre d'une communauté de pratique? Comment suivre une communauté de pratique?

\section{Outil pertinent pour le travail collaboratif}

La communauté de pratique suscite un intérêt grandissant dans divers champs de la santé publique. II s'agit d'un mode de travail collaboratif s'avérant précieux tant pour soutenir les intervenants dans leur pratique que pour faire avancer « la pratique » dans leur champ de savoir. Cela est possible à condition de bien saisir le concept de communauté de pratique, d'en comprendre le fonctionnement et de pourvoir les conditions de succès. La présente fiche d'information a été conçue pour favoriser une représentation partagée de ce qu'est une communauté de pratique et d'enrichir la réflexion des intervenants et gestionnaires de santé publique intéressés à y recourir. La fiche s'appuie sur la littérature, mais est également inspirée des savoirs d'expérience documentés auprès d'informateurs-clés, dans le cadre d'une démarche exploratoire menée par I'INSPQ au printemps 2016 concernant les besoins liés aux communautés de pratique. Les faits saillants de la démarche sont disponibles à l'adresse suivante : https://www.inspq.qc.ca/institut/transfert-desconnaissances/communautes-de-pratique

\section{MESSAGES-CLÉS}

- Au cœur du concept, il y a l'idée d'apprentissage en interaction avec les autres;

- Une communauté de pratique ne se dirige pas, elle s'anime!

- L'efficacité d'une communauté de pratique est liée à son niveau d'activité et d'interaction, à l'engagement mutuel et la satisfaction des membres;

- Une communauté de pratique efficace bénéficie d'un environnement facilitant. 


\section{Communauté de pratique : de quoi parle-t-on?}

Le recours à la communauté de pratique est assez récent dans le réseau de la santé, bien qu'il s'agisse d'une structure de travail collaboratif utilisée dans le monde des affaires depuis plus de 25 ans. Elle y est reconnue pour sa contribution au développement professionnel et à la performance des organisations. La présente fiche illustre comment la communauté de pratique est une stratégie de transfert de connaissances et de développement des compétences adaptée à la pratique de la santé publique.

\section{Définition d'une communauté de pratique}

Le concept s'est surtout fait connaître par le biais des travaux de Lave et Wenger en 1991. Les définitions varient selon les auteurs mais trois dimensions se retrouvent dans la plupart d'entre elles. La définition la plus répandue est la suivante :

"Les communautés de pratique sont des groupes de personnes qui se rassemblent afin de partager et d'apprendre les uns des autres, face à face ou virtuellement. Ils sont tenus ensemble par un intérêt commun dans un champ de savoir et sont conduits par un désir et un besoin de partager des problèmes, des expériences, des modèles, des outils et les meilleures pratiques. Les membres de la communauté approfondissent leurs connaissances en interagissant sur une base continue et à long terme, ils développent ensemble de bonnes pratiques. " (Wenger, McDermott et Snyder, 2002)

La communauté de pratique s'appuierait ainsi sur trois pierres angulaires :

\section{Un domaine d'activité commun}

Constitue le domaine d'activité stratégique ou champ de savoir qui suscite un intérêt commun chez un groupe de personnes. Ce domaine définit ce que ces personnes veulent faire ensemble. C'est la raison d'être de la communauté. Par exemple, l'épidémiologie de terrain, la prévention du jeu pathologique.

\section{Une communauté}

On entend par communauté, la structure sociale qui s'établit au fil des interactions des personnes sur une base régulière et qui conduit à la création d'un sentiment d'appartenance. Associée à l'idée d'intelligence collective (Campos, 2006), elle repose sur l'engagement mutuel des membres et requiert confiance et ouverture. Elle est indissociable de la notion de réciprocité : les membres s'engagent dans une collaboration où ils reçoivent et donnent (Hamel, 2009).

\section{Une pratique partagée}

La pratique réfère aux savoirs scientifiques ou explicites, aux savoirs d'expérience ou tacites, aux outils que les personnes sont intéressées à partager ainsi qu'aux connaissances qu'elles construisent ensemble. La pratique se décline en un répertoire de ressources mis à la disposition de tous les membres. Ce répertoire soutient les membres dans la résolution de problèmes, l'approfondissement de leurs connaissances et le développement de leurs compétences.

Figure 1

\section{Pierres angulaires d'une communauté de pratique}

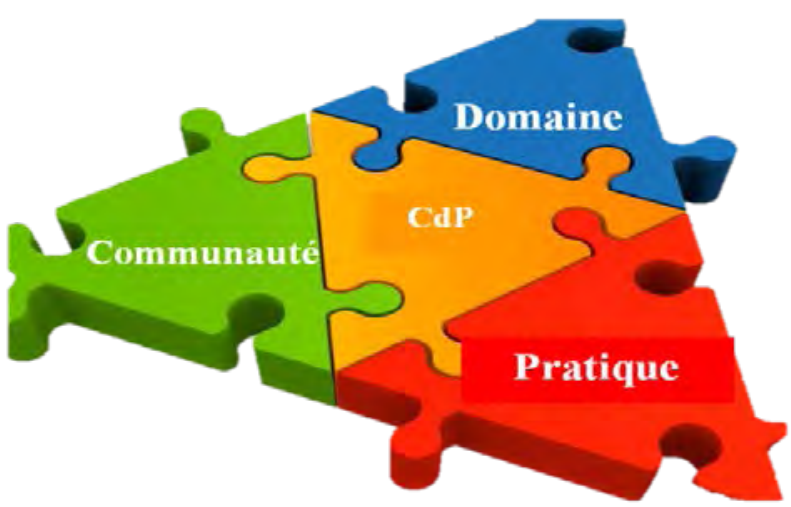

Source : Wenger, McDermott et Snyder, 2002.

La littérature fait état d'un manque de clarté du concept de communauté de pratique notamment au niveau de la complexité des interactions sociales qui y prennent place. Le concept se peaufine donc de plus en plus, notamment dans le secteur de la santé prenant en compte la complexité d'une pratique multidisciplinaire, interorganisationnelle ou même intersectorielle comme c'est le cas en santé publique (Li et al. 2009; Ranmuthagala et al., 2011). 
Au départ, les communautés de pratique émergeaient spontanément des intérêts, passions et problèmes rencontrés par des personnes impliquées dans une pratique spécifique. Peu à peu, les organisations ont reproduit cette stratégie porteuse en mettant en place « intentionnellement » des communautés de pratique pour répondre à des besoins précis. Cela pose toutefois des défis particuliers comme laisser à la communauté de pratique la possibilité de s'autogérer et se structurer tout en la soutenant par l'octroi de ressources et outils requis (Gosselin et al., 2016; Langelier, 2005; CIUSSS CE, 2015).

\section{Pertinence en santé publique}

Les professionnels de santé publique basent leurs pratiques sur des données probantes à travers un processus complexe reposant sur l'intégration judicieuse des connaissances scientifiques, des savoirs d'expérience (tacites), de l'analyse du contexte dans lequel la décision s'applique et des ressources disponibles (Meagher et al., 2012). Des savoirs tacites sont imbriqués à plusieurs des étapes de la planification des actions en santé publique (Khotari et al., 2010).

Les connaissances tacites sont plus intuitives et liées à l'expérience des praticiens (Hautidier, 2006). Comme elles résultent de l'accumulation des connaissances et des expériences pratiques de professionnels n'ayant pas extériorisé leur savoir-faire sous une forme exportable, leur transfert nécessite donc une interaction entre les divers détenteurs de ces savoirs (Lemire et al., 2009).

La communauté de pratique basée sur les interactions entre pairs s'avère ainsi propice d'autant que les connaissances n'y sont pas séparées de leur contexte. Les multiples occasions d'apprentissage prenant place au sein d'une communauté de pratique font en sorte que le recours aux données probantes peut se produire en contexte au moment où le professionnel en a besoin. Les praticiens préfèrent souvent avoir recours aux pairs qui contextualisent l'information plutôt que consulter des ressources documentaires ou en ligne (Meagher-Stuart et al., 2009; Kothari et al., 2010).

Cela est particulièrement vrai lorsqu'ils cherchent une réponse rapide. Lorsqu'ils ont plus de temps, ils consultent une diversité de sources plus explicites: guides de pratique, littérature scientifique, ressources internet, etc.

\section{CONNECTER CE QU'ON SAIT A CE QU'ON FAIT !}

Dans une communauté de pratique, la construction des connaissances s'effectue à travers les discussions et les prises de décisions entre les professionnels (participation) et la réification (verbalisation, description et transcription des savoirs d'expérience dans une forme accessible à tous : présentation faite avec Power Point, rédaction d'une procédure en cas d'éclosion, élaboration d'un outil d'aide à la décision, etc.) (Wenger 1998 dans Davel et Tremblay, 2005; Gosselin et al., 2011.

La communauté de pratique permet le rapprochement entre l'apprentissage (savoir) et l'action (faire).

\section{Des distinctions}

La communauté de pratique se distingue d'autres types de structures de travail du fait que les membres sont mus par le désir et le besoin de partager et d'apprendre les uns des autres sur une base régulière et à long terme. La communauté de pratique met l'accent sur le caractère social et relationnel de la connaissance.

Ainsi une communauté de pratique n'est pas un site WEB, un répertoire des meilleures pratiques, un réseau de diffusion de connaissances, une liste d'abonnées qu'on informe ou un groupe de travail qui réunit des personnes mobilisées autour de l'exécution de tâches menant à un livrable précis avec un échéancier défini dans le temps.

Les réseaux de collaboration entre professionnels se rapprochent du concept de communauté de pratique. La distinction repose sur les intentions d'apprentissage collectif, les moyens mis en place, la régularité des interactions présentes dans une communauté de pratique.

Par ailleurs, un site WEB, un répertoire, des modalités de diffusion de connaissances et des groupes de travail peuvent faire partie des moyens auxquels recourt une communauté de pratique. 


\section{Pourquoi une communauté de pratique?}

La communauté de pratique correspond aux bonnes pratiques en transfert de connaissances : axée sur les besoins des utilisateurs, mise sur l'interaction sociale, mobilise différents types de connaissances, combine diverses stratégies. Pour ces raisons, elle est perçue comme une avenue prometteuse.

La communauté de pratique peut répondre à plusieurs besoins ou objectifs liés à la pratique de la santé publique :

- Accès rapide à de riches sources d'information notamment des données probantes;

- Recours plus facile aux pairs, par exemple en situation d'urgence nécessitant une décision rapide ou lorsqu'il y a incertitude scientifique;

- Accès à l'expertise des membres et experts consultés;

- Partage de pratiques prometteuses, novatrices, expériences, leçons apprises;

- Partage de conseils, avis, idées, ressources, outils, informations;

- Réseautage, établissement plus rapide de liens avec des pairs, réduction de l'isolement;

- Résolution de problèmes;

- Réflexion sur des enjeux communs;

- Coproduction de nouvelles connaissances, génération de nouvelles idées.

La communauté de pratique s'est également révélée utile pour s'approprier de nouvelles lignes directrices, clarifier des notions, harmoniser des façons de faire, se donner une compréhension commune d'un sujet donné, améliorer une situation.
Dans de nombreux champs d'exercice de la santé publique, l'expertise est dispersée dans plusieurs régions du Québec (par exemple : répondants régionaux en ITSS, en prévention du tabagisme, en promotion et prévention en contexte scolaire, etc.). Une communauté de pratique offre la possibilité de regrouper l'expertise de ces acteurs qui autrement :

- Refont les mêmes réflexions dans les différentes régions;

- Dupliquent outils et procédures servant les mêmes objectifs;

- Se sentent isolés.

Dans d'autres champs d'exercice, l'incertitude scientifique liée à l'absence de données requiert le recours aux pairs pour prendre des décisions éclairées. Une communauté de pratique peut permettre aux intervenants d'établir des consensus et de les partager. Des exemples où cela peut s'avérer avantageux :

- En santé au travail : retrait préventif de femmes enceintes dans certaines situations peu connues;

- En santé environnementale : gestion des travailleurs exposés à des contaminants environnementaux.

Dans certains champs d'exercice, des situations d'urgence peuvent survenir et l'expertise collective peut s'avérer précieuse pour prendre des décisions rapidement. Des exemples :

- Gestion des risques en maladies infectieuses (ex. : influenza);

- Gestion des risques en santé environnementale (ex. : déversement de produits toxiques).

Dans certains champs d'exercice, l'expertise est détenue par un nombre limité d'acteurs. La communauté de pratique permet de mettre cette expertise en synergie et de créer une intelligence collective. 


\section{Des bénéfices pour les membres, les organisations et la communauté}

Les membres d'une communauté de pratique peuvent offrir et trouver de l'aide pour les défis auxquels ils font face dans leur pratique.

Autres bénéfices importants documentés :

- Augmentation du sentiment d'identité professionnelle;

- Acquisition et maintien à jour de leurs connaissances;

- Amélioration de leurs compétences professionnelles;

- Augmentation de la confiance dans leur propre expertise;

- Épargne de temps : optimisation de leur temps de recherche;

- Altruisme, se sentir utile, compréhension avantageuse des visions et des idées des autres;

- Satisfaction personnelle à faire partie d'un groupe de personnes intéressantes.

Les organisations peuvent compter sur des professionnels davantage outillés pour aider à leur tour les collègues dans leur équipe, leur service ou leur direction et plus encore :

- Augmentation de la rapidité de transmission des meilleures pratiques;

- Gain de productivité par exemple par le partage de solutions existantes plutôt que de réinventer la roue pour des cas semblables; diminution des risques de duplication;

- Capacité accrue de résolution de problèmes;

- Intégration plus facile des nouveaux dans le domaine;

- Mise à profit de l'expertise des professionnels plus expérimentés;

- Décuplement de l'impact d'une formation grâce à la poursuite des échanges et du partage en communauté;

- Mutualisation des ressources pour accéder à des connaissances rares (Parot et al., 2004);

- Lieu d'expérimentation d'un nouvel outil de travail avant une diffusion plus large.

\section{Quelles formes prennent les communautés} de pratique?

Les caractéristiques de chaque communauté sont uniques en fonction du domaine d'activité, de la pratique concernée et des défis à relever. En santé publique les communautés de pratique existantes ou projetées sont liées soit à une fonction (ex : surveillance), un champ d'exercice (ex. : santé environnementale, maladies infectieuses, promotion et prévention en contexte scolaire) ou une problématique spécifique (ex : jeu pathologique, adaptation aux changements climatiques).

Le mode de création de la communauté, sa composition, la façon dont les membres échangent et quelques autres caractéristiques structurantes définissent une typologie des communautés de pratique (Langelier et al., 2005; Dubé et al., 2006).

\section{Selon le mode de création}

Spontanée : se forme naturellement en raison du besoin de partager des membres.

Intentionnelle : instaurée par une organisation qui a des attentes signifiées.

\section{Selon la composition}

Ouverte : toute personne intéressée peut participer.

Fermée : sélection des membres selon des critères précis comme la profession, le rôle (infirmières, travailleur social, répondant régional, etc.), le champ d'activités (immunisation) ou autres.

Intra ou inter-organisationnelle : selon que la communauté dépasse ou non les frontières d'une organisation (ex. : les directions de santé publique de toute la province ou les professionnels d'un service au MSSS).

Intra ou intersectorielle : selon que les membres appartiennent au même secteur ou à divers secteurs concernés par le domaine.

Les communautés de pratique intersectorielles peuvent permettre une meilleure compréhension des enjeux de santé publique à des partenaires d'autres secteurs détenant les leviers pour agir sur les déterminants de la santé. 
Locale ou dispersée : selon que les membres soient regroupés au sein d'une instance locale ou dispersés dans des zones géographiques différentes (ex. : les gestionnaires de l'INSPQ dans les différentes installations de Québec et Montréal).

Homogène ou diversifiée : selon que les membres appartiennent à des cultures professionnelles semblables ou différentes (ex : différentes professions, différents rôles, différentes provinces, différents secteurs, etc.).

Petite, moyenne ou de grande taille : une communauté peut compter de quelques-uns à plusieurs centaines de membres selon le domaine et les caractéristiques de la communauté. II semble toutefois que plus la communauté est de grande taille, plus les défis sont grands. Certains suggèrent une taille idéale entre 20 et 50 membres pour assurer des échanges productifs et une animation efficace ou la mise en place de sousgroupes d'intérêt dans les communautés de pratique de plus grande taille. Un minimum d'une dizaine de membres est souhaitable pour assurer un volume et une diversité d'échanges.

\section{Selon la façon dont les membres échangent}

Virtuelle : la communauté de pratique utilise uniquement les technologies de l'information et de la communication (TIC) permettant de contrer distance et temps dans ses interactions.

En présentiel : se réunit en face à face.

Hybride : combine les TIC avec des rencontres en présentiel.

Selon la littérature et les personnes interviewées, i est préférable de tenir au moins une rencontre en présence par année pour favoriser l'établissement de liens et la création du sentiment d'appartenance.

Les communautés de pratique en santé publique sont de plus en plus souvent virtuelles offrant l'avantage de réunir des intervenants pour qui il serait difficile autrement de collaborer de façon régulière en raison des contraintes de distance, de temps et d'espace.

\section{Comment fonctionne une communauté de pratique?}

Les membres d'une communauté de pratique se donnent des objectifs en lien avec leur pratique. Ils établissent un programme d'activités permettant les interactions pour partager les connaissances, les utiliser et les produire. Exemples d'activités : ateliers, ouvrage collectif, trousse d'outils, forum de discussion, club de lecture, discussions de cas, webinaires, conférences par un expert invité, activités de réseautage. C'est à travers le dialogue que les connaissances sont exprimées, repérées, identifiées, échangées. Les membres explorent différentes idées, réagissent aux idées des uns et des autres.

Les occasions et les formes d'apprentissage sont multiples mais toutes sont liées à la participation (Parot, 2004) :

- Consulter le répertoire de ressources.

- Réagir, contester, confronter, approfondir.

- Résoudre des problèmes à travers les discussions et autres types d'échanges.

- Solliciter des experts.

Les membres doivent être encouragés à partager des idées, poser des questions, être à l'écoute des problèmes rencontrés par les autres. Ils doivent être conscients qu'ils n'accèdent pas seulement à un service (logique de consommation) mais qu'ils s'engagent dans une relation exigeant une participation active de leur part (Parot, 2004).

II faut ensuite organiser les connaissances partagées entre les membres afin de les rendre facilement accessibles : juste assez (éviter l'amnésie ou au contraire "le documentalisme » à outrance), juste à temps (rendre l'information disponible au moment où elle est nécessaire) (Langelier, 2005; CIUSSS CE, 2015). II s'agit de mettre en contact rapidement un individu avec la source de connaissances qu'il lui faut. Une bonne pratique est d'utiliser plusieurs logiques de classement afin de simplifier l'accès aux connaissances (ex. : par thème, par sujet, par auteur, par date, etc. La constitution d'un annuaire avec des rubriques comme "Qui fait quoi? Qui a fait quoi? Qui sait quoi? 
Qui connaît qui? » est très utile. La façon de gérer la documentation dans le répertoire partagé constitue un des moyens de gagner la confiance des participants (Langelier, 2005).

\section{Évolution d'une communauté de pratique}

Selon Wenger, une communauté de pratique évolue à travers cinq stades différents (Wenger et al., 2002).

\section{Figure 2. Les stades d'évolution d'une} communauté de pratique

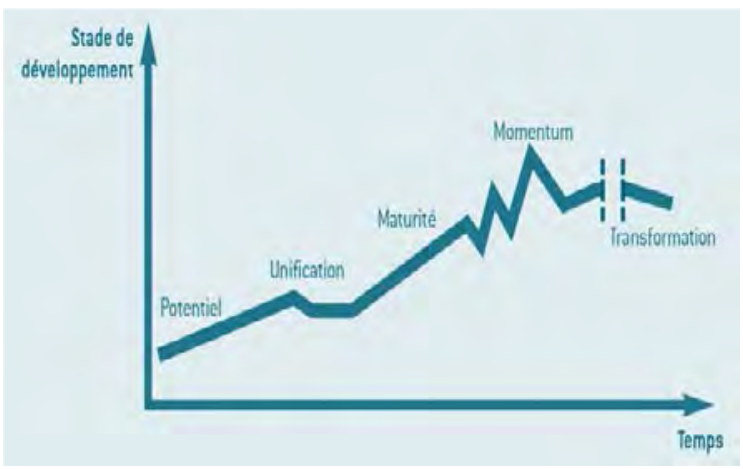

Tirée de CEFRIO (2005). Travailler, apprendre et collaborer en réseau, p.22 (Adaptation Richard McDermott, 2002)

\section{LES CINQ STADES}

Potentiel : les gens se découvrent les uns et les autres, imaginent diverses possibilités.

Unification : adhésion d'une masse critique de personnes engagées dans la communauté de pratique. Augmentation des connaissances partagées.

Maturité : la communauté de pratique développe sa vitalité, les activités pas nécessairement nombreuses sont intenses.

Momentum : correspond à la période de productivité : échanges féconds, activités, productions.

Transformation : point tournant : poursuivre? changer?

Toute communauté de pratique évolue à son rythme selon ses caractéristiques. Plusieurs mois sont nécessaires pour que la communauté de pratique atteigne un niveau où elle devient efficace, porte fruits. II faut donc s'attendre à une période moins productive avant de recueillir les avantages et les bénéfices. La communauté de pratique doit être soutenue car elle est fragile pendant l'évolution vers la productivité.
«Une communauté de pratique se construit ensemble et traverse diverses étapes. II faut du temps pour que la confiance s'établisse et que les liens se tissent. "

Citation captée d'une entrevue réalisée dans le cadre d'une démarche exploratoire menée par l'INSPQ. Document « La communauté de pratique : quels sont les besoins des acteurs en santé publique? disponible ici : https://www.inspq.qc.ca/institut/transfert-desconnaissances/communautes-de-pratique

\section{Types de relations}

Il se développe un niveau et une qualité d'interaction variables au sein des communautés de pratique selon le champ d'exercice et les objectifs poursuivis. Cela se traduit par une variation dans le type de rencontres, leur fréquence ainsi que les modalités d'échange et fréquence des interactions entre les rencontres.

Interactions possibles et pertinentes (Langelier, 2005; Tétreault, 2013; Parot et al., 2004; Leclerc-Jacques, 2013; Ford et al., 2015, ASSSM, 2010) :

- Faire un appel à tous,

- Répondre à une question posée par un membre,

- Partager une information connue jugée utile,

- Contribuer à une production collective,

- Prodiguer un conseil,

- Émettre un commentaire,

- Faire une recommandation,

- Présenter une expérience probante,

- Présenter un élément de connaissance robuste en lien avec le domaine d'intérêt.

Tous les membres ne participent pas au même niveau. Plusieurs études rapportent que le profil de participation habituel est le suivant : les contributeurs qui se répartissent entre un noyau très actif (environ $10 \%$ ) insufflant énergie et dynamisme à la communauté) et un noyau actif ( $20 \%$ des membres); les observateurs légitimes majoritairement silencieux représentant environ $70 \%$ des membres qui demeurent en périphérie. Ceux-ci bénéficient toutefois des échanges et peuvent éventuellement jouer un rôle plus actif (réservoir de contributeurs potentiels) (Leclerc-Jacques, 2013; Tétreault, 2013). II faut aussi noter que des actions peuvent être entreprises suite à la lecture d'une discussion tant chez les contributeurs que chez les 
observateurs. Certains observateurs transmettent à d'autres ce qu'ils ont lu (Ford et al., 2015).

Le défi est de faire progresser les membres de la périphérie vers les plus actifs ou très actifs sans oublier que les membres transfèrent d'un groupe à un autre selon les sujets abordés. II y a intérêt à valoriser chaque contribution.

Le niveau d'interaction peut également être décrit en termes de coprésence, de collaboration et de coopération (Campos, 2006) :

- Coprésence : on se sert de la communauté de pratique principalement pour se réseauter. Le niveau d'interactions est très faible. On met en commun des documents, des outils de travail, etc.

- Coopération : on participe à la communauté lorsqu'on cherche à obtenir une réponse à une question. Le niveau d'interactions est plus élevé mais les échanges demeurent dans le pratico-pratique. On est davantage axé sur SA pratique.

- Collaboration : on profite de la communauté pour réfléchir sur la pratique, partager des expériences et développer les meilleures pratiques dans son domaine. On est axé sur LA pratique.

Des relations de pouvoir peuvent apparaitre et appellent à la vigilance pour s'assurer que les décisions et productions de la communauté de pratique s'appuient sur les évidences et non les opinions des leaders ( $\mathrm{Li}$ et al., 2009; Bertone et al., 2013).

Dans le réseau de santé publique, les membres d'une communauté de pratique n'ont habituellement pas de contacts quotidiens, peuvent appartenir à plusieurs organisations et sont souvent impliqués à divers degrés dans la pratique constituant le domaine d'intérêt de la communauté. (ex. : membres régionaux impliqués dans plusieurs dossiers en maladies infectieuses: ITSS, immunisation, gestion des risques, etc.).

\section{Les facteurs influençant la participation}

Plusieurs facteurs sont susceptibles d'influencer le partage de connaissances au sein d'une communauté de pratique (Lafleur, 2012; Leclerc-Jacques, 2013; Hamel, 2009; Harvey, 2011; Bertone et al., 2013).

\section{FACTEURS $\uparrow$ LA PARTICIPATION}

- Confiance mutuelle chez les membres : la communauté de pratique doit être un espace sécurisant faisant en sorte que les membres s'expriment librement. Ils ne doivent pas craindre le jugement ou l'embarras;

- Confiance que les connaissances partagées sont fiables;

- Pertinence des discussions pour la pratique;

- Infrastructure technologique facile à utiliser.

\section{FACTEURS $\downarrow$ LA PARTICIPATION}

- Crainte de ne pas être pertinent;

- Crainte de perdre son pouvoir en le partageant;

- Impression de moins offrir d'informations que d'en retirer;

- Manque de temps (selon les priorités du membre et pas nécessairement par manque de temps accordé par les supérieurs);

- Culture ne favorisant pas le partage des connaissances;

- Perception qu'il s'agit d'un travail supplémentaire;

- Manque de réponses ou délai trop long pour répondre aux questions posées.

\section{Les facteurs de succès à réunir}

\section{Facteurs de succès liés à l'organisation}

En santé publique, la plupart des communautés de pratique réunissent des membres provenant de diverses organisations (ex: communauté de pratique en maladies infectieuses réunissant des professionnels du MSSS, des directions de santé publique et des CISSS/CIUSSS). Chacune des organisations est concernée par la mise en place des facteurs de succès et exigera une concertation entre les décideurs (Létourneau, 2010). 
L'aspect organisationnel constitue un élément prépondérant dans l'équation du succès. Les facteurs les plus déterminants sont:

- Le degré de formalisme institutionnel de la communauté de pratique dans l'organisation (ou les organisations du réseau de santé publique par exemple).

C'est-à-dire lui permettre de prendre place comme structure légitime. Selon Wenger la communauté de pratique peut aller d'invisible à institutionnalisée en passant par marginale, autorisée ou soutenue. Ce degré de formalisme aide à justifier le temps que les professionnels consacrent à la communauté.

\section{- L'allocation de temps pour soutenir une animation} de qualité.

Les exigences pour l'animation varient toutefois selon la typologie de la communauté notamment en ce qui concerne la fréquence des activités interactives à mettre en place (ex : discussion de cas, webinaires, etc.). En santé publique, dans la plupart des champs d'exercice, les pratiques des intervenants évoluent à un rythme qui ne requiert pas des activités interactives hebdomadaires entre les membres. C'est toutefois le cas dans les champs d'exercice où les connaissances évoluent rapidement ou dans lequel les situations professionnelles peuvent confronter les acteurs à des interrogations fréquentes (quotidiennes, hebdomadaires ou mensuelles) notamment en santé au travail, en maladies infectieuses et en santé environnementale. C'est aussi le cas lorsqu'un champ de pratique est en émergence. Cependant, peu importe le champ d'exercice, une animation sur une base régulière est nécessaire pour permettre de développer le sentiment d'appartenance, le réflexe de suivre les activités de la communauté de pratique et maintenir l'intérêt des membres.

- L'allocation de temps reconnu dans la tâche des membres pour leur participation à la communauté de pratique afin qu'ils puissent réaliser des apprentissages significatifs.

La participation équivaut en moyenne entre 1 et $3 \mathrm{~h}$ par semaine (interrogations quotidiennes dans la pratique) ou 0,5 à 1,5 jour par mois dans les domaines d'activité suscitant moins d'interrogations quotidiennes ou hebdomadaires.
Il est de moins en moins fréquent de trouver des organisations accordant du temps pour examiner une pratique, en discuter avec des collègues et s'approprier celle-ci (Harvey, 2011). Ces précieux moments doivent être ajoutés de manière intentionnelle. Sans temps disponible, chaque individu passe de projet en projet sans avoir la possibilité de réfléchir aux leçons à tirer.

\section{- Un leadership affirmé}

Plusieurs communautés de pratique existantes ou envisagées en santé publique sont interorganisationnelles et requièrent que le leadership de collaboration en communauté soit présent et visible dans chacune des organisations concernées (Harvey, 2011).

Les communications doivent être harmonisées auprès de chaque organisation et devraient faire ressortir les performances des communautés.

Les communautés de pratique doivent être intégrées au niveau le plus haut de la stratégie des organisations (Harvey, 2011).

\section{- Une reconnaissance de la participation}

Les organisations doivent inciter leurs professionnels à participer en réduisant les barrières et en valorisant les apprentissages réalisés.

" Il est important que les membres puissent être en mesure de dégager du temps pour participer activement à la communauté de pratique. Pour ce faire, ils doivent percevoir que le temps, les efforts de partage de leurs connaissances et l'énergie qu'ils investissent dans la communauté sont valorisés par l'organisation. II faut joindre le geste à la parole! » (CEFRIO, 2005).

- Le parrainage par un gestionnaire influent.

- Le soutien à la formation des animateurs et membres pour fonctionner en communauté de pratique.

- Le soutien technologique : plateforme collaborative, moyens de communication à distance en temps réel (synchrone) ou en temps différé (asynchrone), outils de gestion documentaire; etc. 
II est important de former les membres de la communauté de pratique à l'utilisation de la plateforme choisie, mais aussi sur ce qu'est une communauté de pratique, le partage des connaissances, la communication interpersonnelle à distance, la résolution de problèmes, etc.

\section{Dans le cas de communautés de pratique intentionnelles}

- S'assurer de thématiques, tâches et attentes porteuses et stimulantes pour les membres tout en étant d'intérêt stratégique pour les décideurs et gestionnaires;

- Laisser à la communauté de pratique le soin de s'auto-organiser.

\section{Facteurs de succès liés aux membres}

Disposant d'un environnement favorable, les membres d'une communauté de pratique doivent mettre l'épaule à la roue pour assurer un fonctionnement efficace. Les facteurs individuels importants sont :

- L'ouverture aux idées nouvelles;

- La curiosité;

- L'autodiscipline;

- La souplesse;

- L'initiative, l'intérêt;

- La participation qui constitue le principal enjeu. Les membres doivent se donner le temps.

Il est souvent plus important de savoir aider et se faire aider que d'être en mesure de répondre soi-même à toutes les questions. Afficher un sens critique tout en étant constructif. Être en mesure d'identifier ses propres lacunes et besoins (Langelier, 2005; Lafleur, 2012).

La communauté doit trouver son rythme c'est-à-dire une fréquence adéquate pour les activités afin qu'elle ne soit ne soit pas trop accaparante mais demeure stimulante. II faut aussi développer des espaces où les membres peuvent communiquer entre eux entre les rencontres (Gosselin, 2011).

\section{EFFICACITÉ $=$ IMPLICATION ${ }^{\curlyvee}$ X ÉCHANGES ${ }^{\Upsilon}$}

Les membres doivent se donner du temps et inscrire une participation systématique à la communauté dans leur agenda. Par exemple, contribuer à un échange, consulter ses collègues, répondre à une question, transmettre une information, partager une expérience (Jacob et Paquet, 2013).

\section{Facteurs de succès liés à la communauté}

Plusieurs personnes interviewées dans le cadre de la démarche menée par l'INSPQ confirment que les membres doivent rapidement voir la valeur ajoutée de partager leurs expériences, idées, connaissances. Ils doivent saisir l'utilité que cela aura pour eux, pour la pratique dans leur domaine.

« Il faut que la communauté de pratique soit proche du quotidien des membres. Plus on est proche de ce qu'ils souhaitent, de ce qu'ils veulent, mieux ça fonctionne ".

Citation captée d'une entrevue réalisée dans le cadre d'une démarche exploratoire menée par l'INSPQ portant sur les besoins liés à la mise en œuvre d'une communauté de pratique en santé publique. Document "La communauté de pratique : quels sont les besoins des acteurs en santé publique? disponible ici :

https://www.inspq.qc.ca/institut/transfert-desconnaissances/communautes-de-pratique

Les autres facteurs importants sont :

- Des objectifs bien définis.

- L'engagement mutuel sans lequel la confiance ne peut s'établir. Les membres doivent se sentir en confiance de partager leurs connaissances et utiliser celles des autres.

- Des objets de travail en lien direct avec la pratique des membres.

- Une animation de qualité : importance d'avoir des animateurs dédiés. Comme les membres d'une communauté de pratique ne sont pas actifs en permanence, il faut que l'animateur soit proactif afin de soutenir la participation. Fournir une information régulière, des nouvelles, un courrier récapitulatif des échanges, etc. (Parot et al. 2004; Langelier, 2005; Jacob et Paquet, 2013).

- Des activités répondant aux besoins des membres (satisfaction des membres). 


\section{Quelles sont les étapes de mise} en œuvre?

Au moment d'initier une communauté de pratique, certaines étapes sont requises. Ces étapes ne représentent pas un processus linéaire et peuvent se chevaucher ou être interverties selon les contextes.

\section{Constitution d'un groupe noyau et} préparation

Que la communauté de pratique soit spontanée ou intentionnelle, il est judicieux de former un groupe «noyau » pour préparer le projet de communauté. Ce groupe devrait regrouper des individus motivés : initiateur/fondateur, parrain, expert de contenu, spécialiste des TIC, etc. II peut parfois être bénéfique d'adjoindre un conseiller expérimenté.

Figure 3 Étapes de mise en oeuvre d'une communauté de pratique

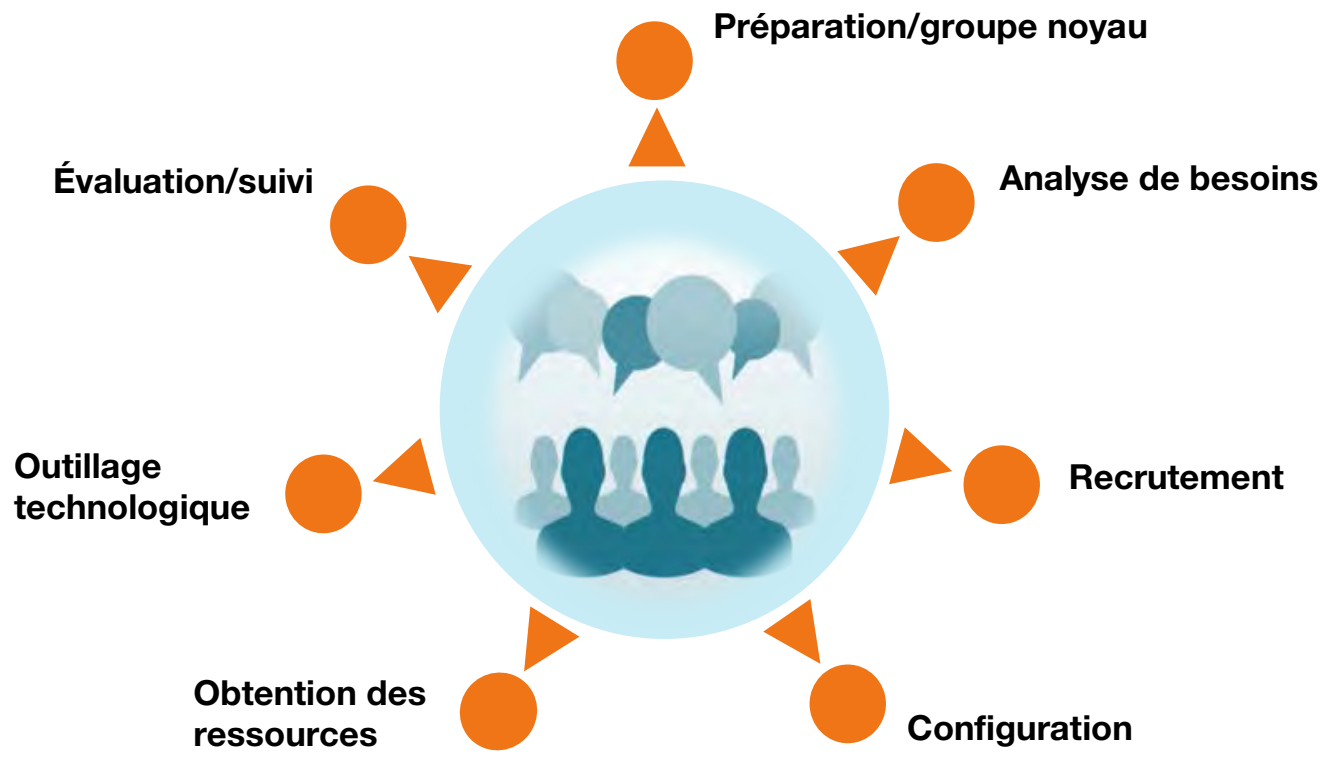

La majorité des initiateurs/fondateurs de communauté de pratique interrogés par I'INSPQ ont recherché et obtenu le soutien d'une personne plus expérimentée lors du démarrage de leur communauté. De plus, cela s'est avéré un besoin exprimé par $100 \%$ des professionnels qui envisagent la mise sur pied d'une communauté de pratique. ressources 
Le groupe «noyau » se penche sur les objectifs de la communauté de pratique, le profil des membres à recruter, les caractéristiques souhaitées, etc. Le tout étant évidemment modulable après validation auprès des futurs membres.

\section{Analyse des besoins}

Il faut identifier les besoins précis qui conduisent à choisir ce mode de travail collaboratif et déterminer à quel (s) groupe (s) de professionnels la communauté sera utile.

Diverses modalités sont possibles, le sondage en ligne s'avérant le plus souvent utilisé.

\section{Recrutement}

C'est souvent le domaine d'intérêt qui influence le recrutement des membres. Dans le cas d'une communauté de pratique intentionnelle, les attentes détermineront parfois qui recruter. Un réseau existant peut s'avérer un bon point de départ. La diversité culturelle (ex : divers secteurs d'activités, champs d'exercice ou régions) favorise des échanges riches tout en posant un défi pour établir la confiance et la poursuite d'objectifs communs.

Selon une étude du CEFRIO, les volontaires sont plus susceptibles de participer activement que les personnes qui ont été forcées d'intégrer la communauté de pratique, ce qui a été corroboré auprès des personnes interrogées.

\section{Configuration de la communauté}

La configuration signifie définir les paramètres de la communauté de pratique de façon à répondre aux besoins des membres ainsi qu'aux attentes de l'organisation dans le cas d'une communauté de pratique intentionnelle.

La communauté de pratique s'organise selon ses besoins et son contexte. Voici les actions proposées :

- Attribuer des rôles et responsabilités. Une même personne peut jouer plusieurs rôles ou plusieurs personnes peuvent se partager un même rôle (ex. : animation «tournante » entre quelques personnes).
LES DIVERS ROLES POSSIBLES (ADAPTÉ DE LANGELIER, 2005 ET JACOB ET PAQUET, 2013)

Animateur : assure le leadership, organise, soutient les relations interpersonnelles, stimule les échanges, motive, relance, fait le lien avec les collaborateurs externes. Peut déléguer certaines de ces tâches...et en assumer d'autres également (ex. : administrateur).

Membre : Adhère à la communauté et participe aux activités qui y sont organisées. Doit s'impliquer et participer au bon fonctionnement.

Fondateur : Initie la communauté. Participe à l'élaboration de la mission et des objectifs.

Expert de contenu : Détient une expertise spécifique d'intérêt pour la communauté. Aide, enrichit, valide.

Veilleur : Détecte et analyse le contenu thématique.

Administrateur : Organise et coordonne les événements et activités de la communauté.

Personne-ressource en TI : Surveille et entretient les outils de collaboration. Adapte les outils selon les besoins : paramétrage; gestion des droits et accès; sécurité.

Mentor : membre expérimenté chargé de l'intégration des nouveaux membres; explique les normes et politiques de la communauté.

Rapporteur : Identifie et publie sous forme de documents, les présentations, rapports, savoirs pertinents, meilleures pratiques, nouvelles approches ou leçons tirées de la communauté.

Parrain : Veille à la visibilité de la communauté, ses ressources et son importance stratégique. Protecteur de la communauté lui offrant une reconnaissance.

Il est important que l'attribution des rôles et responsabilités soit vue comme une forme de reconnaissance et non comme une charge supplémentaire (Harvey, 2011). 


\section{Établir une charte de participation à la} communauté avec les futurs membres s'avère judicieux pour établir clairement les principes de collaboration. La charte permet notamment de déterminer comment les membres seront recrutés, les valeurs, les engagements à respecter, les objectifs poursuivis, les ressources partagées, les modalités de fonctionnement, les rôles et responsabilités à assumer, l'utilisation qui sera faite des fruits de la communauté, etc. La signature de la charte formalise l'engagement des membres. La charte facilite l'implication de nouveaux acteurs. Des exemples de charte de communauté sont disponibles ici :

https://www.inspq.qc.ca/institut/transfert-desconnaissances/communautes-de-pratique

\section{Obtention des ressources}

Les ressources requises varient d'une communauté de pratique à l'autre. II n'est pas nécessaire de disposer d'un important budget, il faut surtout du temps.

\section{Principaux investissements :}

- Ressources humaines : participation des animateurs, groupe noyau, membres; formation animateurs, formation membres sur le travail en communauté de pratique;

- Ressources technologiques : plateforme collaborative, développement informatique, licences, hébergement, formation à l'utilisation;

- Frais de déplacement et de communication.

\section{Outillage technologique}

II existe une panoplie d'outils technologiques permettant de décupler la portée d'une communauté de pratique en éliminant les contraintes de temps, de distance et d'espace. Diverses technologies permettent de maintenir les interactions que ce soit pour discuter, échanger ou collaborer.

Toutefois, il ne faut pas perdre de vue que la technologie est en soutien à l'engagement et l'implication des membres.
"La technologie doit être au service de la communauté et non son essence. Toutefois elle ne doit pas non plus devenir un obstacle à son bon fonctionnement. ”

Citation captée d'une entrevue réalisée dans le cadre d'une démarche exploratoire menée par l'INSPQ. Document « La communauté de pratique : quels sont les besoins des acteurs en santé publique? disponible ici : https://www.inspq.qc.ca/institut/transfert-desconnaissances/communautes-de-pratique

Les outils disponibles permettent les types d'interactions suivants :

- Synchrones : même moment/même endroit ou endroits différents;

- Asynchrones : moments différents/même endroit ou endroits différents.

Les principales fonctionnalités disponibles sont :

- Création de groupes avec accès différenciés;

- Forums de discussion;

- WIKI, blogues;

- Bibliothèques, répertoire d'outils et documents;

- Moteur de recherche;

- Système de notification;

- Visioconférence;

- Téléconférence;

- Babillard électronique.

La technologie actuelle offre des plateformes intégrant plusieurs de ces fonctionnalités (ex : Sharepoint, Liferay, etc.). Les caractéristiques de la plateforme (ex : sa convivialité, sa facilité d'utilisation, sa rapidité, sa capacité d'intégration avec d'autres logiciels) et les fonctionnalités souhaitées par les membres (ex : système de notification, bibliothèque, suivi des versions d'un document élaboré collectivement) sont autant de critères à considérer.

Il faut s'assurer de la compatibilité avec l'environnement technologique des membres. L'accessibilité sur divers supports (ordinateur, cellulaire, tablette) constitue un atout. Les outils existants dans une organisation ou un réseau peuvent s'avérer un bon point de départ (ex : site internet existant avec zone de dépôt de documents et d'échanges). 
QUALITÉS RECHERCHÉES DANS LA TECHNOLOGIE (LANGELIER, 2005; LAFLEUR, 2012; ARZUMANYAN, 2016)

Adaptée aux besoins des membres;

Simple, accessible, conviviale et compatible;

Évolutive car les besoins changent sans cesse;

Sécuritaire et confidentielle;

Abordable : faibles coûts (développement, entretien, hébergement).

Il est à noter que certaines de ces ressources peuvent être gratuites (ex: plateforme collaborative) ou déjà couvertes par l'organisation (ex: licences de téléconférence / vidéoconférences, salle de rencontre). Dans le réseau de santé publique, des défis sont rencontrés : sécurité de l'information empêchant le recours à des plateformes gratuites; bande passante parfois faible dans certaines régions; etc.

\section{À propos de la diffusion...}

Selon le contexte, une communauté de pratique choisit de s'afficher publiquement ou non. Dans certaines communautés, il peut s'avérer important de la faire connaître de façon attrayante en période de recrutement auprès de potentiels membres.

La communauté de pratique doit décider si elle se donne une identité visuelle et réfléchir sur la façon dont elle souhaite partager les produits de la communauté.

Au moment du démarrage, la première rencontre officialise le début des activités de la communauté de pratique. Autant que faire se peut, cette réunion devrait avoir lieu en face à face pour susciter la confiance et créer des liens. Cette importante première fois pose les jalons d'une collaboration future que l'on souhaite dynamique et stimulante. L'importance des rencontres en face à face est reconnue dans la littérature comme critère de réussite des communautés en ligne (Tétreault, 2013).

Dans le réseau de santé publique, il faut composer avec une importante limitation des déplacements. II faut alors faire preuve de créativité pour soutenir la création de liens malgré la distance.

\section{À PROPOS DE L'ANIMATION...}

«Une communauté de pratique ne se dirige pas, elle s'anime... »

(Livre blanc « Les communautés de pratique »).

Les personnes interviewées dans le cadre de la démarche de l'INSPQ ont corroboré les données de la littérature quant au rôle crucial de l'animation qui constitue le plus grand défi d'une communauté de pratique. Les interactions entre les membres sont soutenues par des activités régulières allant de l'échange d'idées en réunion ou dans un forum de discussion, à la collaboration en sous-groupes, à des événements spéciaux ponctuant la vie de la communauté.

L'animateur d'une communauté de pratique doit avoir la reconnaissance de ses pairs et posséder certaines qualités personnelles notamment, le sens de l'écoute, l'empathie, le dynamisme et l'aisance dans les relations interpersonnelles.

II ne doit pas nécessairement être un expert du contenu mais il doit posséder des habiletés pour effectuer les tâches inhérentes à une communauté de pratique (ex : planification, communication, préparation de l'espace de collaboration, recrutement des membres, animation des activités, suivi de la communauté, organisation des connaissances partagées et documents, etc.). II faut être conscient qu'animer une communauté de pratique virtuelle c'est autre chose qu'animer en face à face !

Enfin tel que mentionné dans les facteurs de succès, l'animation requiert du temps. Un volet plus complet sur l'animation d'une communauté de pratique sera développé dans une prochaine fiche. 


\section{Comment suivre une}

\section{communauté et en mesurer l'efficacité?}

Les retombées potentielles d'une communauté de pratique peuvent être d'ordre individuel (membre), organisationnel ou collectif (communauté). Ces retombées sont liées à un fonctionnement efficace et dynamique de la communauté de pratique qui à son tour, repose sur un environnement facilitant. II peut donc s'avérer pertinent de documenter la mise en œuvre avant de se prononcer sur les impacts.

Comme les communautés de pratique prennent du temps à évoluer et devenir productives, les activités doivent être soutenues au fil du temps. L'évaluation doit être dynamique, réalisée à intervalles réguliers et se pencher sur différents éléments adaptés au stade de développement de la communauté.

Malgré le grand intérêt théorique et pratique pour les communautés de pratique, il y a peu de propositions basées sur des preuves pour les évaluer. La capacité d'attribuer les changements à une communauté de pratique est limité dans les études réalisées à ce jour (Ranmuthugala et al., 2010; Bertone et al., 2013 ; Richard et al., 2014). Toutefois le nombre d'articles scientifiques qui se penchent sur ces aspects est en augmentation. Des auteurs proposent des cadres d'évaluation comportant des dimensions et indicateurs qui reflètent les divers aspects des communautés de pratique et les processus y ayant cours (Ranmuthugala et al., 2010; Wenger et al., 2011; Bertone et al., 2013 ; Richard et al., 2014).

Il est recommandé d'avoir recours à des indicateurs tant quantitatifs que qualitatifs afin de pouvoir rattacher l'action de la communauté à des résultats opérationnels. Une combinaison de méthodes est suggérée afin de suivre l'évolution de la communauté de pratique, sa « santé » et son efficacité.

\section{Suivre l'évolution de la communauté}

Parmi les moyens permettant de suivre la communauté de pratique au fil de son évolution afin d'apporter des ajustements notons :

- Tenir un journal de bord;

- Prévoir des rencontres entre l'animateur et le parrain ou le gestionnaire responsable.

\section{Mesurer la «santé » de la communauté de pratique}

La « santé " de la communauté de pratique est associée aux processus et activités au sein de la communauté et inclut la :

- vitalité : niveau d'activité et d'interaction,

- satisfaction des membres.

L'analyse des statistiques de fréquentation permet de documenter le niveau d'activité (ex : nombre de visites sur la plate-forme, sections visitées, nombres d'échanges, etc. Pour ce qui est de la satisfaction des membres on aura plutôt recours à des sondages en ligne, focus group ou entrevues. Des aspects comme la réponse aux besoins des membres, les activités les plus pertinentes, les difficultés rencontrées peuvent être documentées, etc.

\section{Mesurer l'efficacité de la communauté de pratique}

L'efficacité d'une communauté de pratique réfère à son impact (Jacob et Paquet, 2013) et inclut :

- L'atteinte d'objectifs fixés ou attentes signifiées;

- Les apprentissages réalisés par les membres;

- La valeur créée pour l'organisation ou le réseau de santé publique incidemment (par exemple la réduction du temps de recherche d'informations par les intervenants).

Des sondages pré/post sur les compétences professionnelles et les collaborations, focus group et entrevues s'avèrent de bons moyens pour aller documenter des éléments comme ce que les membres ont adapté dans leur pratique, ce qui leur a permis de résoudre certains problèmes, etc. 


\section{En résumé}

Par sa structure et son fonctionnement, la communauté de pratique s'avère une stratégie des plus pertinentes pour lier la théorie à la pratique et contribuer à l'intégration des données probantes (savoirs tacites et explicites) dans les prises de décision en santé publique. La communauté de pratique représente toutefois un changement de culture posant un défi à la fois aux professionnels et aux organisations. Pour les individus, il s'agit de passer d'une logique individualiste à une logique de partage. Pour les organisations, il s'agit d'évoluer vers des environnements moins contrôlés où plus de latitude est consentie aux professionnels et une plus grande valorisation est accordée au savoir et à l'apprentissage. Cela suppose un effort d'ouverture. Pour espérer tirer profit de la communauté de pratique il faut réunir les facteurs de succès reconnus. Dynamiser les communautés, les aider à se structurer et à fonctionner efficacement prend du temps et exige des ressources.

Le champ d'études des communautés de pratique dans le secteur de la santé et plus spécifiquement en santé publique est encore jeune. D'autres expériences et études sont requises afin de documenter la façon dont les apprentissages y sont réalisés et les processus par lesquels les communautés produisent leurs effets.

Le plus difficile avec une communauté de pratique ne serait pas tant sa mise en place mais bien d'assurer sa pérennité !

POUR EN SAVOIR PLUS...

Vous trouverez des éléments complémentaires sur le site de l'INSPQ à l'adresse suivante :

https://www.inspq.qc.ca/institut/transfert-desconnaissances/communautes-de-pratique

\section{Principales références}

Agence de la santé et des services sociaux de la Montérégie. (2010). L'animation d'une communauté de pratique: une compétence incontournable au courtage des connaissances. Bulletin de Veille VlsAge.

Arzumanyan, L., \& Mayrhofer, U. (2016). L'adoption des outils numériques dans les communautés de pratique: Le cas du Groupe SEB. Revue Française de Gestion, 42(254), 147-162.

https://doi.org/10.3166/rfg.2016.00006

Bertone, M., Meessen, B., Clarysse, G., Hercot, D., Kelley, A., Kafando, Y., ... Witter, S. (2013). Assessing communities of practice in health policy: a conceptual framework as a first step towards empirical research. Health Research Policy and Systems, 11, 39. https://doi.org/10.1186/1478-4505-11-39

Campos, M. N. (2006). Des communautés de pratique aux communautés épistémiques. In Communautés virtuelles: penser et agir en réseaux. Québec: Les Presses de l'Université Laval.

Centre de réadaptation en dépendance de MontréalInstitut universitaire. (2015). Les communautés de pratiques: définition, enjeux et spécificités dans le monde médical. Recension des écrits. CIUSSS du Centre-Est-de- l'île-de-Montréal.

Dube, L., Bourhis, A., \& Jacob, R. (2006). Towards a typology of virtual communities of practice. Interdisciplinary Journal of Information, Knowledge and Management, 1, 69.

Ford, J. R., Korjonen, H., Keswani, A., \& Hughes, E. (2015). Virtual communities of practice: can they support the prevention agenda in public health? Online Journal of Public Health Informatics, 7(2). https://doi.org/10.5210/ojphi.v7i2.6031

Hamel, C. (2009). Determinants of participation in an online community of practice (Thesis submitted in partial fullfilment of the requirements Masters of sciences in E-business technologies). Université d'Ottawa, Ottawa, Ont. Retrieved from http://www.ruor.uottawa.ca/handle/10393/28383

Harvey, J.-F. (2010). Comment favoriser le partage des connaissances? Le cas des communautés de pratique pilotées. Gestion, Vol.35(4), 73. https://doi.org/10.3917/riges.354.0073 
Stenuit Hautdidier, F. (2006). Créer et animer des communautés de pratique: préconisations pour une entreprise de formation et de conseil (Thèse en vue de l'obtention du DESS en Sciences de l'information et de la documentation spécialisés). Conservatoire national des arts et métiers, Institut national des techniques de la documentation, Paris. Retrieved from

http://memsic.ccsd.cnrs.fr/mem 00000413/document

Jacob, R., \& Paquet, M.-J. (2013). Mettre en oeuvre une communauté de pratique. Principes et facteurs de succès. présenté au Séminaire de formation. Formation des cadres et dirigeants de HEC Montréal, Montréal (Québec).

Kothari, A., Rudman, D., Dobbins, M., Rouse, M., Sibbald, S., \& Edwards, N. (2012). The use of tacit and explicit knowledge in public health: a qualitative study. Implementation Science, 7(1).

https://doi.org/10.1186/1748-5908-7-20

Lafleur, C. (2012, Octobre). La participation des membres au sein de la Communauté virtuelle de pratique en amélioration continue (Essai présenté à la Faculté d'administration en vue de l'obtention du grade de Maître en administration, programme de Management Public). Université de Sherbrooke, Sherbrooke (Québec).

Langelier, L., Wenger, E., White, N., Smith, J. D., \& rowe, K. (2005). Travailler, apprendre et collaborer en réseau. Montréal: CEFRIO.

Lemire, N., Souffez, K., \& Laurendeau, M.-C. (2009). Animer un processus de transfert des connaissances. Bilan des connaissances et outil d'animation. [Montréal]: Direction de la recherche, formation et développement, Institut national de santé publique Québec.

Langelier, L., Wenger, E., White, N., Smith, J. D., \& rowe, K. (2005). Travailler, apprendre et collaborer en réseau. Montréal: CEFRIO.

Leclerc-Jacques, B. (2013, t). Une communauté de pratique en adaptation aux changements climatiques: pour une meilleure gestion des connaissances en santé environnementale? (Essai présenté dans le cadre du M.B.A. en responsabilité sociale et environnementale des organisations. Essai-stage). Faculté des sciences de l'administration, Université Laval, Québec (Québec).

Létourneau, P., Beaudoin, J., Shabah, Y., \& Girard, M. (2010). Comment gérer des communautés de pratique interorganisationnelles? Gestion, Vol.35(4), 64. https://doi.org/10.3917/riges.354.0064
Li, L. C., Grimshaw, J. M., Nielsen, C., Judd, M., Coyte, P. C., \& Graham, I. D. (2009). Evolution of Wenger's concept of community of practice. Implementation Science, 4(1), 11. https://doi.org/10.1186/1748-59084-11

Li, L. C., Grimshaw, J. M., Nielsen, C., Judd, M., Coyte, P. C., \& Graham, I. D. (2009). Use of communities of practice in business and health care sectors: a systematic review. Implementation Science: IS, 4, 27. https://doi.org/10.1186/1748-5908-4-27

Meagher-Stewart, D., Solberg, S. M., Warner, G., MacDonald, J.-A., McPherson, C., \& Seaman, P. (2012). Understanding the Role of Communities of Practice in Evidence-Informed Decision Making in Public Health. Qualitative Health Research, 22(6), 723-739. https://doi.org/10.1177/1049732312438967

Parot, S., Talhi, F., Monin, J.-M., \& Sebal, T. (2004). Livre Blanc. Les communautés de pratique. Analyse d'une nouvelle forme d'organisation \& panorama des bonnes pratiques, nov. 2004. France: Knowings et le Pôle Productique Rhône-Alpes.

Ranmuthugala, G., Plumb, J. J., Cunningham, F. C., Georgiou, A., Westbrook, J. I., \& Braithwaite, J. (2011). How and why are communities of practice established in the healthcare sector? A systematic review of the literature. BMC Health Services Research, 11, 273. https://doi.org/10.1186/14726963-11-273

Richard, L., Chiocchio, F., Tremblay, M.-C., Lamy, G., Champagne, F., \& Beaudet, N. (2014). Communities of practice as a professional and organisational development strategy in local public health organizations in Quebec, Canada: an evaluation model. Health Policy, 9(3), 26-39.

Wenger, E., McDermott, R. A., \& Snyder, W. M. (2002). Cultivating communities of practice: a guide to managing knowledge. Boston, Massachussets: Harvard Business School Press.

Wenger, E., Trayner, B., \& De Laat, M. (2011). Promoting and assessing value creation in communities and networks: a conceptual framework (No. 18). Netherlands: Ruud de Moor Centrum. 


\section{La communauté de pratique un outil pertinent : résumé des connaissances adaptées au contexte de la santé publique}

\section{AUTEURE}

Lyne Arcand, médecin-conseil

Vice-présidence à la valorisation scientifique et aux communications

\section{EN COLLABORATION AVEC}

Karine Souffez, experte en transfert de connaissances

Vice-présidence à la valorisation scientifique et aux communications

\section{MISE EN PAGE}

Hélène Fillion, adjointe à la direction

Vice-présidence à la valorisation scientifique et aux communications
Ce document est disponible intégralement en format électronique (PDF) sur le site Web de l'Institut national de santé publique du Québec au :

http://www.inspq.qc.ca.

Les reproductions à des fins d'étude privée ou de recherche sont autorisées en vertu de l'article 29 de la Loi sur le droit d'auteur. Toute autre utilisation doit faire l'objet d'une autorisation du gouvernement du Québec qui détient les droits exclusifs de propriété intellectuelle sur ce document. Cette autorisation peut être obtenue en formulant une demande au guichet central du Service de la gestion des droits d'auteur des Publications du Québec à l'aide d'un formulaire en ligne accessible à l'adresse suivante :

http://www.droitauteur.gouv.qc.ca/autorisation.php, ou en écrivant un courriel à : droit.auteur@cspq.gouv.qc.ca.

Les données contenues dans le document peuvent être citées, à condition d'en mentionner la source.

Dépôt légal - $1^{\text {er }}$ trimestre 2018

Bibliothèque et Archives nationales du Québec

ISBN : 978-2-550-80539-7 (PDF)

๑ Gouvernement du Québec (2018)

$\mathrm{N}^{\circ}$ de publication 2351

Institut national 